\title{
Fazit und Ausblick in die Zukunft
}

Die Ausführungen haben gezeigt, dass in der Antarktis sehr spezifische Bedingungen vorzufinden sind. Angefangen bei der geringen Besiedelung und technologisch-infrastrukturell geringen Erschließung, über den speziellen rechtlichen Status hin zu den langen, komplexen Versorgungsrouten. Hinzu kommen die auch kurzfristig kaum planbaren Witterungsbedingungen, die die antarktische Logistik immer wieder vor große, teils unüberwindbare, Herausforderungen stellt.

Auch wenn eine umfassende Besiedelung kaum zu erwarten ist, ist doch sowohl bei den touristischen Aktivitäten als auch bei den Forschungsbestrebungen zumindest ein gewisser Zuwachs zu erwarten. Während in der Tourismusbranche gestiegene Mobilität im Zuge der Globalisierung dafür verantwortlich sein dürfte, so spielt die Antarktis aufgrund ihrer „Unberührtheit" eine wichtige Rolle bei der Erforschung weltweiter Klimaphänomene. Ein Abschmelzen der antarktischen Eisflächen würde angesichts ihres Anteils an weltweiten Wasservorkommen zudem eine deutliche Erhöhung des Meeresspiegels nach sich ziehen. So erscheint es umso wichtiger, die strengen Umweltauflagen in der Region zu halten, wenn nicht gar auszubauen. Auch eine Begrenzung des Tourismus als Beitrag zum Erhalt der Region wird immer wieder diskutiert.

Langfristig aber dürfte die Frage entscheidend sein, ob es gelingt, den nationalen Interessen an einer kommerziellen Nutzung des „weißen Kontinents“ auch bei der nächsten Revision des „Antarctic Treaty“ wieder Einhalt zu gebieten zu können. Sollte dies nicht gelingen, so dürften sich mit der Ausbeutung der vermuteten Rohstoffvorkommen umfassende Erweiterungsanforderungen an eine „,antarktische Logistik“ stellen. Spätestens dann würde klar, dass neben den formulierten Handlungsempfehlungen für eine internationale Logistik auch die Antarktis selbst von steigender, auch kommerzieller Bedeutung für Dienstleister werden könnte. 